\title{
Biomedical Nanotoxicology and Concerns with Environment: A Prospective Approach for Merger with Green Chemistry Enabled Physicochemical Characterization
}

\author{
Parth Malik', Tapan K Mukherjee ${ }^{2}$ and Man Singh ${ }^{1,3 *}$
}

${ }^{1}$ Centre for Nano Sciences, Central University of Gujarat, Gandhinagar, India

${ }^{2}$ Department of Biotechnology, Maharishi Markandeshwar University, Mullana (Ambala), Haryana, India

${ }^{3}$ School of Chemical Sciences, Central University of Gujarat, Gandhinagar, India

\begin{abstract}
Of late, nanotechnology has emerged to be a very reliable bridge in trying to club salient fields of natural sciences on a common platform. Deservedly enough, nanomaterials have enabled numerous unconventional wonders to be accomplished through their incorporation, either on a singular or on complexed form. However, the rapid strides being accomplished on a tremendously high speed have sheerly ignored some ground realities in terms of ethical considerations. Bestowed with unconventional physicochemical properties and material behavior, nanomaterials are often surprise candidates in terms of their interaction with several other materials. This leads to a significant difficulty in the discard of integrated structures or individual materials carrying nanomaterials. The emerging threat of nanotoxicology from interactions thereof has attracted significant attention of environmental conservationists from the different corners of the world. In terms of physicochemical behavior, it is extremely essential to have a sound knowledge of the nanomaterial behavior and probable risk assessment. With this background, this review article sheds light on the behavioral aspects of nanomaterials with a comprehensive recalling of the case studies. The propositions of novel invention from Indian soil, Survismeter, friccohesity and tentropy have been made for further strengthening the risk assessment of nanomaterials.
\end{abstract}

Keywords: Nanomaterials; Physicochemical; Bestowed; Propositions; Tentropy

\section{Nanotoxicology: An Introduction}

Nanotoxicology can be defined as the study of toxic effects emanating from the use of nanomaterials. Nanomaterials are the unique materials having any one of the dimensions typically falling within the range of 1-100 $\mathrm{nm}$. They have highly different properties and behavior from the bulk material due to their characteristically small dimensions. They have a high surface area to volume ratios and due to this the physical phenomenon associated with them are highly unconventional. The dominance of quantum effect begins to occupy the forefront. Nanomaterials even made up of inert materials like gold or silver etc. have very unusual properties in the sense that they have high reactivity and are very differently enabled for different applications, which are not even visible at the bulk scale. Nanotoxicology can broadly be said to be the science of engineered nanodevices and nanostructures that deals with the effects in the living organisms. The field of nanotoxicology explores the effects of exposure to nanomaterials. Foremost significant thing about nanotoxicology is to have a sound understanding of nanomaterial sources in the environment. It is a misconception that only the laboratory experiments and highly sophisticated, target oriented activities lead to the generation of nanomaterials. There are also a significant degree of natural sources of nanomaterials, such as those of pollen grains, the small, ultrafine particles in smoke, air contaminants and so many other natural exhaust mechanisms carrying nanomaterials to a higher or lower extent. Nanotoxicological studies are intended at determining whether and to what extent these properties may pose a threat to the environment [1]. For instance, it has been found that diesel nanoparticles are very injurious to health in the mice genome and result in cardiovascular complication [2]. With the rapid and tremendous strides encountered by science and technology in almost every day to day task of life and the ever dwindling shrinkage and pressure mounting on the different energy resources of the nature; the nanotechnological innovations have entered almost every scientific and technical requirement of the society. To determine the toxicity of nanomaterials, assays need to be developed that involve the dispersion of these nanomaterials in the different media. However, this may result in the appearance of particular kind of toxicities depending on the nature of surrounding media involved. Determination of toxicity of nanomaterials in different stages of food chains must be surveyed and estimated in order to obtain a more appropriate picture of the effects of the nanomaterials on environment as well as human health.

As a consequence, we must consider about the ethical implications of the use of nanomaterials and the different pros and cons associated with it. This is the chief reason for the increasing and stimulating impetus being received by nanotoxicology in different countries of the world. The expert committees set up by so many developed economies of the world like the Environmental Protection Agency (EPA) of USA, the Expert Committee for Research on Nanomaterials in USA and The Royal Society of Nanomaterials in United Kingdom (UK); all are ample proofs of the fact that nanomaterials are a different class of materials even as waste products and deserve special attention and that too as early as possible. For understanding the concept of nanotoxicology in detail, two things are very essential to have an insight upon. First is the

*Corresponding author: Professor Man Singh, School of Chemical Sciences Central University of Gujarat, Gandhinagar, India, Fax: +91-079-232-60076; Tel: +91-079-232-60210; E-mail: mansingh50@hotmail.com; parthmalik1986@gmail.com

Received July 18, 2014; Accepted September 16, 2014; Published September 23, 2014

Citation: Malik P, Mukherjee TK, Singh M (2014) Biomedical Nanotoxicology and Concerns with Environment: A Prospective Approach for Merger with Green Chemistry Enabled Physicochemical Characterization. J Microb Biochem Technol S9: 001. doi:10.4172/1948-5948.S9-001

Copyright: ( 2014 Malik P, et al. This is an open-access article distributed under the terms of the Creative Commons Attribution License, which permits unrestricted use, distribution, and reproduction in any medium, provided the original author and source are credited 
interaction of the nanomaterials with the environmental components and the second is the interaction of different nanomaterials with the living systems. To dig further deeper in this direction, we must be aware that a nanomaterial is very small and has the rapid tendency to randomly move, get combined with a number of other different kinds of materials and segregate under the influence of environmental fluctuations of physical conditions. The text ahead mentions the nature of nanomaterials that are threatening to a sustainable development of nature and the interaction of nanomaterials with the environment in almost every feasible manner.

\section{Engineered nanomaterials: The core of nanotoxicology}

Engineered Nanomaterials (ENMs) are those materials which are intentionally designed with a view to enhance the performance in several technological and industrial scale processes [3]. These do not exist naturally and are thoroughly man-made in their background. This is why they show the nanoscale effects in an uncharacteristic manner. This feature makes them special and comparatively special agents for contributing to the toxicity of nature. These nanomaterials exhibit the nanoscale phenomenon in an extraordinary manner. For example, the number of atoms lying on the surface in these nanomaterials is uncharacteristically high. This makes them behave differently even when compared to the normal nanomaterials. Due to large number of surface atoms, the engineered nanomaterials occupy more matter over the same volume when compared with their bulk scale counterparts [4]. Owing to this, these materials exhibit stronger reactivities and higher tendencies to get reduced by combining with other materials. This further makes it more suspicious to give rise to toxicity multiplying mechanisms. Engineered nanomaterials are basically of two types, viz. carbon based or non-carbon based; accordingly categorized as being organic or inorganic in nature. Therefore, it is highly essential to first understand the variable nature of these ENMs in an elaborative manner for gaining a sound knowledge of the corresponding interaction mechanisms propagated by them under the physiological as well as environmental conditions.

The critical parameters include their interaction with the components of environment, their tendency to get biodegraded and bio accumulate in nature. An important aspect of the engineered nanomaterials is their tendency to get agglomerated and in the liquid media. They form aggregates which are random in nature and enter the food chain through aquatic organisms. They persist more in the sea water and this tendency further results in exhibition of novel effects as the behavior of aggregated complexes will be significantly different from those of the individual nanoparticles. These properties and aspects of engineered nanomaterials present a concrete evidence of the environmental threats which they can potentially lead to. This in turn makes the characterization of the engineered nanomaterials highly urgent and it also necessitates that the synthesis process should be manipulated in such a manner that a better control over the properties and the potential risks for environmental deterioration can be exercised. For instance, carbon nanotubes can be prepared by different methods and these are significantly different in terms of their chemical and physical behavior and as a result exhibit highly diverse ecotoxicological properties (Figure 1) [5-8].

\section{Diversified Interaction Patterns of Nanomaterials}

With the technological breakthroughs enabled by the optimized application of different nanotechnological interventions, there seems to be a wide gap prevailing between a proper understanding of the potential threats manifested in the technological wonders of this technology

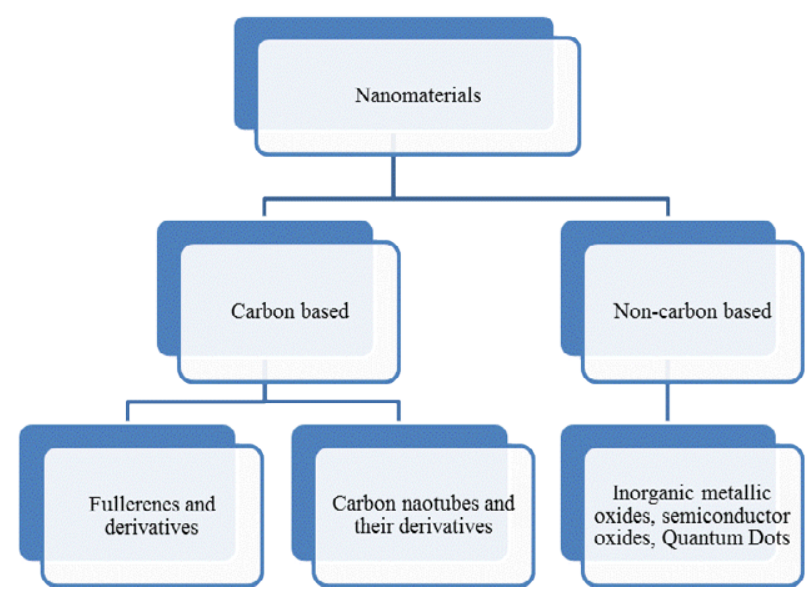

Figure 1: The diverse categorization of nanomaterials on the basis of their hierarchical makeup.

with reference to damage of environment and health [9-12]. As this technology centers around miniaturization, so it often results either in the wastage or the requirements of highly sophisticated inputs; going by the very basic structural and designing aspects of the nanostructures. Off course, this does not cover the related unintentionally created random nanomaterials. However, nanomaterials, inherently due to their small size; pose some serious health problems. For example, they go unnoticed by the barriers (e.g. skin) of human systems. This is further rendered difficult to be borne, owing to the functionalization possessed and surface modification of the overall molecular arrangements. In some situations, the hydrophicity incorporated becomes the surprising facilitator by virtue of the lipidic solubility of these carriers [13-15].

\section{Habitat mediated impacts of nanomaterials}

Aerial interactions: Air seems to be very first and obvious environmental zone through which a material exerts its environmental effect. The behavior of nanoparticles in the air is influenced by several factors, which, in turn, have a strong influence on their chemical and physical aspects. The major reasons for the nanomaterial initiated problems seem to be the consequences of the respective time duration for which nanomaterials remain suspended in air, the effect of gravitation and the corresponding sedimentation rates which, in turn, is dependent on their size and respective chemical compositions. The resulting aspects of the nanoscale hazards are manifested in their combining capacity and the inherent attribute of getting agglomerated to give rise to complex geometries and morphologies. However, almost all of these factors seem to be very precisely modulated through the fluctuations in the environmental conditions. As an instance, it is very well known that the composition of air is highly variable in different places of the world, which renders the toxic effects to be differently expressed in humid atmospheres and coastal areas, those in unfamiliar conditions being far more vulnerable [16-18]. Even though, the procedures for gathering all complex and minute details regarding the chemical behavior of the ultrafine particles are well understood and established [19], yet whether all these analyzing procedures are applicable to study nanomaterials with reference to their key physicochemical properties, is significantly unconfirmed till date. Interestingly, nearly all of these complications seem to be build up on each other, primarily due to the highly diminishing dimensions of nanomaterials which manifest themselves in their rapid escaping tendencies and they remain unnoticed even after sufficient time lapses. 
For instance, the specially coated metal oxide nanoparticles are often engineered to alter their surface behavior and kinetic aspects. These modifications significantly alter their behavior and resultant chemical and physical interactions.

At or around the nanoscale, the size regime has a sharp bearing on the aerial behavior of the nanoparticles. The rate of molecular diffusion of the particles is normally inversely proportional to the diameter while that of the gravitational settling varies directly with the mean diameter of the particles. Particles with diameters less than 80 nanometers are highly small and they have the tendency to agglomerate. However, these get settled due to gravitational impacts and are therefore not significantly harmful. Similar is the story with nanomaterials of diameters greater than 2000 nanometers. However, the chief culprits are the particles of intermediate size. They have their diameters varying from 100 to several thousands of nanometers. These have a tendency of getting accumulated and remaining suspended in the air for the longest durations. Further, their removable can be accomplished only after deposition on specific surfaces which is a slow, gradual and a specifically monitored process [20].

Terrestrial interactions: Soil is characterized in terms of terrestrial ecosystems, the largest geographical domain contributing to the biodiversity. The ultrafine and highly small scale dimensions of nanomaterials tend to further complicate the interactions of nanomaterials with soil. The behavior of nanomaterials as toxicants via their interactions with soil is extremely tough to be analyzed because of highly diverse nature of soil particles in so many different chemical and physical forms. The interactions of nanoparticles with nanomaterials are highly different and unpredictable in different soil textures such as that of soil media, soil organic matter, soil solutions and soil pre-existing wastes. Owing to their unprecedented and unexpected chemical properties, the nanomaterials really present a very gloomy picture in terms of their handling, transport and toxic effects. Their interactions with different forms of soil are further highly different. To add to this the significantly different nature of soil components has a very important role in further transport of nanomaterials. The most surprising and daunting task is encountered when the nanopollutants and wastes combine with organic materials present in the soil. This makes their morphology even harsher; thereby amplifying the associated environmental threats [21]. The deposition and settling of nanoparticles within the larger soil particles and then the bioaccumulation via entry through food chains in animals and plants has been of considerable concern. Due to their highly small size, nanomaterials get absorbed by soil particles and ultimately move faster than larger particles. This leads to the eventual entrapment of the soil particles inside the soil matrix. However, this is not always the case since this absorption is influenced by the critical physical features of size, chemistry, surface functionalization and the corresponding mode of preparation. Numerous attempts have been made towards the estimation of the relative motions of the entangled and absorbed nanomaterials and the study of insoluble particles in terms of their behavior at the nanoscale [22-24]. The interaction of nanomaterials with the soil is also critically influenced by the nature of the soil. This is particularly interesting with respect to the study of the interactions of colloidal mineral nanoparticles with the soil. This is because these particles are charged due to their reactive nature and physical methods of formation which ultimately adversely affects their gravitational settling in the soil concerned [25].

To add to this are the interesting surface properties of the nanomaterials. Nanoparticles are photoactive and catalytically active in nature $[26,27]$. When these particles are entangled with soil particles, upon incidence of sunlight, they form complexes and aggregates with the soil particles. This further amplifies their segregation and settling abilities, leading to critical consequences as nanoparticles get absorbed by soil and are consequently ingested by plants. From here, they enter the food chains and manifest themselves as threatening interferers to the ecosystem. The behavior of soil which has absorbed nanoparticles is also conventionally very different. They are not even biodegraded due to their complex chemical nature and have a tendency of persistence. To sum up, we can say that the minute size of the nanomaterials accompanied by complex surface behaviors are the most potent threats to the environment.

In humans, the nanoparticles of metal oxides have been shown to cause oxidative damage by stimulating the generation of free radicals. Studies have reported the toxic effects of the polystyrene nanoparticles on the blood cells, which acutely affect the mechanism of thrombogenesis. Similarly, significant threats for the transition metal oxides to human lung epithelial systems have also been reported. Metallic oxides such as those of zinc oxides, titanium oxides, cerium oxides have been found to cause serious damage on the endosomes with the concurrent generation of Reactive Oxygen Species (ROS) [28-30]. In a concurrent sense, Copper Oxide $(\mathrm{CuO})$ nanoparticles have been found to cause some of the most threatening toxic effects, resulting in significant oxidative damage to DNA through the stimulation of oxidative lesions [31,32]. The eventual fate of the particular kind of nanoparticles in a particular terrestrial environment is determined not only by the corresponding physical and chemical properties. This is owing to the fact that after being present in soil; individual nanoparticles become soil entities and attain relatively more complex surface morphologies [33]. In fact, the nature of a particular soil also has a significant influence in deciding the ultimate fate of the nanomaterials in the environment. Various soil factors including those of $\mathrm{pH}$, ionic strength, zeta potential and soil texture seem to be of paramount concern in deciding the eventual fate of the nanoparticles being released as wastes in a particular kind of habitat [34-36]. In plants, nanoparticles of Zinc Oxide $(\mathrm{ZnO})$ in particular, have been reported to induce toxicity and subsequent inhibitory effects on growth. As far as transport of nanomaterials through terrestrial organisms is concerned, it has been reported that $C$. elegans is a nematode that is actively involved in contributing towards the toxicity and bioaccumulation of nanoparticulate metallic oxides. An active uptake of $\mathrm{ZnO}, \mathrm{Al}_{2} \mathrm{O}_{3}, \mathrm{TiO}_{2}$ and $\mathrm{Ag}$ nanoparticles by C. elegans has been reported to be a vital cause for decreasing reproduction and inhibition of growth $[37,38]$.

Aquatic interactions: Aquatic nanotoxicology is rapidly assuming significant proportions in the domain of toxicity evaluation of nanoscale wastes. This is inherently due to the different types of interactions of nanomaterials with water than those which are exhibited, when they are not in the range of nanoscale dimensions. The interactions of nanoparticles with water remain the key to evaluate their corresponding aquatic toxicities. Aquatic nanoparticles encompass not only the particles that are present in large scaled water reservoirs such as those of seas, oceans and lakes but also which remain suspended in air and come to earth in the form of rain. In general, it has been realized that these nanoparticles settle at a far lower pace than their terrestrial and aerial counterparts, due to the exertion of up thrust in liquids. These aerial nanoparticles, due to their exceptional photo-labile attributes, interact with sunlight while traversing with rain, as a result of which complication with biotic and abiotic components of environment further complicates the eventual fate of nanomaterials. Along the surface, the abiotic degradation occurs 
which dominates the exposure to sunlight. These photochemically induced reactions are the governing factors for environmental fate of nanoscale toxicologies of these particles. As a consequence of these reactions, physical and chemical properties of the nanomaterials get significantly altered, eventually manifesting in the form of their altered and unpredictable behavior in the water bodies. This behavior may also include the risks to aquatic biodiversity and deterioration of aquatic habitat. As far as aquatic toxicity of the nanomaterials is concerned, prevailing literature reports suggest that aquatic invertebrates are the most vulnerable organisms of threats since they traditionally suffer the most via environmental contaminants [39,40]. Studies with nanomaterial toxicities in aquatic organisms have reported the corresponding genotoxic as well as ecotoxic effects. In this direction, a number of worthy research efforts have reported the harmful impacts of transition and semi-metallic oxides like those of cerium dioxide, silicon dioxide and titanium dioxide on the various living aspects of the aquatic organisms in significant detail. Interestingly, cerium dioxide $\left(\mathrm{CeO}_{2}\right)$ nanoparticles have been found to be associated and responsible for DNA damage in most of the aquatic organisms when evaluated. The combined effect of silicon dioxide and cerium dioxide nanoparticles on the aquatic organisms has led to the finding that both these inorganic metallic oxides increase the mortalities in aquatic organisms. In particular with $D$. magna species, a significant risk for DNA damage and the reduction in the reproduction capacity was found in the members exposed to cerium dioxide nanoparticles [41]. Other than this, a number of threats have been reported attributing to the discharge of silver nanoparticles in aquatic bodies. Nanoparticles of silver have been found to induce edema production, abnormalities in the spine, fins, heart, brain and eyes have been reported; providing significant information that nanoparticles of silver are highly toxic to aquatic life [42].

To summarize, we can say that the complex interactions between nanomaterials and the biosphere makes the nanomaterials very tough customers, which can have extremely deleterious effects on the biodiversity possessed. The resultant toxic interactions culminate in not only the nature getting more and more contamination and vulnerable for infection but also makes the surrounding environment far more risk prone to their threats.

\section{Interaction of nanomaterials on the human systems}

The significant risks of nanomaterials in humans have been mainly postulated to mature through interactions with skin, respiratory and gastrointestinal tracts. Following are the brief consequences of the minute interactions involved:

Interaction with skin: Skin is the outermost layer of the body that interacts with any foreign material. Skin is normally composed of three distinct layers, viz. epidermis, dermis and subcutaneous layer. The outer layer of the epidermis, also known as Stratum Corneum (SC) covers the entire outside surface of the body and the layer is rich in keratinized tissues. In routine, skin comes in contact with several toxic materials of diverse backgrounds. However, it is the presence of keratin rich regions which prohibit the entry of toxic materials across the inner layers of the skin. The SC layer is mostly composed of dead cells and is keratin rich in nature. The morphology of skin tissues is different throughout the body. This is due to the fact that the outermost epithelium tissue is prevalent in different shapes across the different body tissues and these shapes are mostly due to functional distinctions. Most of the mammals have their skin covered with small hair like follicles. These hairs make the entry of most minute materials restricted through the passage across them. The studies based on interaction of foreign materials with the skin have mostly focused on the potential analysis of the drug delivery vehicles being commonly used. These include the solid-lipid nanoparticles, microscale emulsions, titanium dioxide micro and nanosized particles and some liposomal preparation on the microscale (Figure 2).

The easiest and most susceptible route for the interaction of nanomaterials with the skin is through the application of tropical creams and several other drug administration routes [43-46]. For instance, the use of silver nanoparticles for the surgical dressings and the bandaging of wounded parts have been suggested but it is not beneficial in entirety and there are still some ethical concerns which pose a matter of serious debate and concern over their acceptance. In case of titanium dioxide nanoparticles, studies have shown that these nanoparticles can easily overcome the keratin barriers of the human skin and can reach even the epidermis and dermis. The strong interactions of the titanium dioxide nanoparticles, which are almost regular ingredients of most of the sunscreen creams and the conventionally used body lotions, with the internal layers of the skin, are highly critical for the further growth and development of new skin cells. Not only this, these nanoparticles can even induce mutations at the genetic levels through this exposure route. The problems of skin contraction and those of allergies are frequent in persons prone to the use of sunscreens and skin care cosmetics. The concerned risks assume even more severity when we talk about the heterogeneous nanomaterials with highly diverse physicochemical properties and surface morphologies. Studies have shown that these particles can cross the entire SC layer and get deposited in the dermal and epidermal tissues. In a related study involving the treatment of skin burns with silver nanoparticle coatings, it has been found that such patients suffer from abnormal levels of silver in their bloodstreams and due to this they suffer from a number of skin related problems. The most popular are adverse coloration of the skin and the irritating allergies [47]. Similarly, pertinent toxicities have been reported with fullerene based nanopeptides, having high penetration power and getting propelled by outer skin layers into the dermis [48]. Quantum dots and other carbon based structures have been shown to interfere with lymphatic systems and the other lymph nodes in the body, leading to diverse health conditions $[49,50]$.

Thus, in all we can say that interactions of nanomaterials with the skin are highly complex and pose significant risks that are priorities to be analyzed.

Interaction with respiratory tract: Respiratory system is the second fundamental physiological compartment, after the mouth of the living systems that an inhaled particle encounters after gaining entry into the body. With no gravitational affects dominating, the dynamic flow regimes and size-dependent physicochemical behavior

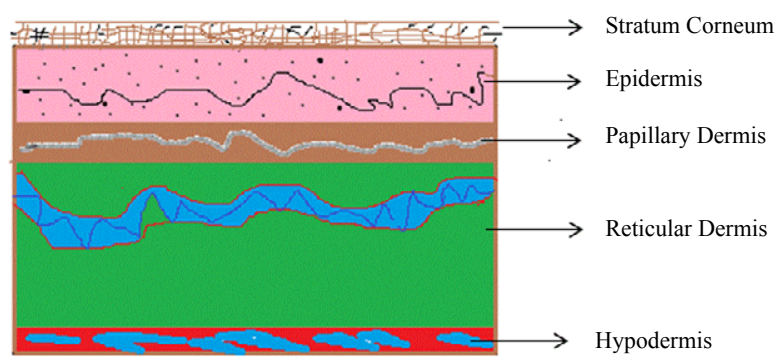

Figure 2: An overview of internal morphology of skin. 
of nanomaterials; there is a significant risk amplifying and augmenting the toxicity of nano-pollutants, upon being inhaled via the process of respiration. However, due to subtle differences in the size, chemical composition, surface morphology and particle dynamics, the behavior of nano-pollutants inside the living systems is extremely unpredictable. This makes the hazards from nanomaterials even more vulnerable. The nano-dimensional particles behave very differently from their normal counterparts of higher size limit due to which these can't be retained or restricted and are eventually washed away with the upper airway pathways of the muco-ciliary systems. Particles less than 2.5 micrometers in size can go down and finally get deposited in the alveolar sacs. The ultrafine particles below the dimensions of 100 nanometers have a tendency to get deposited in the alveolar region [51,52].

Eventually after their absorption through lung epithelium, nanoparticulate impurities travel to the blood and lymph and ultimately invade the lymph nodes, spleen, bone marrow and cardiac system components. The respiration mediated toxicity of the nanodimensional particulate matter is specially considered under the terminology of pulmonary toxicity and is affected by a number of parameters other than the particle size. These factors are as mentioned ahead:

1. Concentration and the size distribution of the particles in the final formulation

2. Dosage of such particles with respect to a particular tissue.

3. Surface charges on particles.

4. The degree to which the particular particles agglomerate or coalesce.

5. Method of synthesis of the particles involved, which may vary from solid-phase or liquid-phase synthesis of the overall particle formulation and the overall surface modification.

6. Surface treatment of the particles with respect to their applications, particularly in the case of engineered nanoparticles.

The invasion upto this much extent by the ultrafine particles ultimately, leads to the coagulation and obstructed blood flow which inhibits the overall coordination in the body (Table 1) [70-72]. Studies have proved that there is bio-persistence of silver nanoparticle based impurities once they gain entry into the bodies through respiration. They have been shown to persist in alveolar macrophages for nearly seven days and found as precariously toxic to alveolar macrophages as well as lung epithelium tissues [72,73].

Interactions with gastrointestinal tract: Nanomaterials can reach to the gastrointestinal tract either after getting muco-ciliary clearance from the upper respiratory layers or directly through ingestion of food, water, drugs and drug delivery devices. The research investigations showering light over the toxic effects of nanoparticles over the gastrointestinal locations in the living organisms remain hardly numbered. Toxicity of copper at the nanoscale in mice has been reported and it has been shown that copper when assimilated in the nanoscale results in the pathological damage to liver, kidney and spleen [74]. Similarly, the occurrence of skin discoloration has been reported with the possible reason being the absorption of nanoscale colloidal silver from the intestinal tract [75]. Fluorescent labeled polystyrene nanoparticles have also been reported to be prevailing in intestinal lymphatic tissues, and observed to be severely implicated in Peyer's Patches [76].

\section{Detection and Analysis of Environmental Nanoparticles}

Nanomaterials in the environment (air, soil and water) are very tough candidates to be analyzed for, not only due to their uncharacteristically small size but also due to uncertain physical and chemical interactions with other materials in the environment. A number of sophisticated as well as extremely fundamental microscopic and spectroscopic techniques are currently employed for the detection of nanomaterials in the environment. These include the scanning and transmission electron microscopies, the confocal and phase contrast microscopic analysis and the structural investigation tools of nuclear magnetic and infra-red spectroscopies along with their specified variants to elucidate the respective elemental compositions. One specific aspect of all these characterization techniques is that each of these requires unique sample preparation. Moreover, in general, the toxicity of nanomaterials is an implicit tendency of engineered nanoparticles. This is so because in their native forms, nanomaterials are already present in a significant proportion and are well confronted with respect to minimize their toxicities. Intentionally designed or engineered nanomaterials sometimes possess some additional materials on their surfaces which make them tough candidates for characterization. Interestingly, in some situations, nanoparticles are deliberately deposited on the surface to impart the specific functionalities, even though they are not at all harmful. This signifies that nanomaterials are not risk posing entities in all the configurations. Thus an appropriate distinction in between functionally useful and potentially toxic nanoparticles is needed to be made so as to clearly understand and confront the likelihood threats.

\begin{tabular}{|c|c|c|c|}
\hline Nanoparticle Type & Source & Potential Risks Involved & References \\
\hline Silver nanoparticles & $\begin{array}{l}\text { Mining activities, industrial activities, } \\
\text { incineration of biomedical wastes }\end{array}$ & $\begin{array}{c}\text { Oxidative stress, apoptosis, necrosis of cells, } \\
\text { altered cell signaling. }\end{array}$ & [53-55] \\
\hline Titanium dioxide nanoparticles & $\begin{array}{l}\text { Produced as pigments used in paints, } \\
\text { coatings, plastics, inks, foods, cosmetic } \\
\text { products, pills etc. }\end{array}$ & Oxidative stress, retarded cell growth & [56-58] \\
\hline $\begin{array}{l}\text { Carbon based nanoparticles: fullerenes, } \\
\text { carbon black and carbon nanotubes }\end{array}$ & $\begin{array}{l}\text { Byproducts of poly aromatic hydrocarbons } \\
\text { formation, celestial activities, natural and } \\
\text { anthropogenic sources }\end{array}$ & $\begin{array}{l}\text { Retarded cell growth, decreased cell viability, } \\
\text { oxidative stress and apoptosis }\end{array}$ & [59-61] \\
\hline $\begin{array}{l}\text { Molybdenum, iron oxide, aluminium Oxide } \\
\text { and cerium oxide and Silicon based } \\
\text { nanoparticles }\end{array}$ & $\begin{array}{l}\text { Automobile fuels, mining and metallurgical } \\
\text { operations, research activities, dust storms etc. }\end{array}$ & $\begin{array}{l}\text { Oxidative stress, cytotoxicity, hemolysis and } \\
\text { cell death }\end{array}$ & [62-66] \\
\hline Polymeric nanoparticles & $\begin{array}{l}\text { Biomedical applications, conjugated assay } \\
\text { research }\end{array}$ & $\begin{array}{l}\text { Oxidative Stress, inflammation, alteration in } \\
\text { cellular morphology and functioning }\end{array}$ & [67] \\
\hline $\begin{array}{l}\text { Heterogeneous nanostructures: quantum } \\
\text { dots, nanodevices etc. }\end{array}$ & $\begin{array}{c}\text { Diagnostic assays, sensing materials, probes, } \\
\text { advanced detecting methods }\end{array}$ & $\begin{array}{l}\text { Chromatin condensation, free radical } \\
\text { formation, arrest of cell growth and } \\
\text { sometimes even cell death }\end{array}$ & {$[68,69]$} \\
\hline
\end{tabular}

Table 1: An overview of mainly employed nanoparticles with their potential sources and toxicities. 
Toxic nanoparticles or nanomaterials may include nanoparticles generated by burning of fossil fuels, wastewater treatment processes, drainage seepage problems or from soil-erosion and metallurgical complicacies. The key parameters for characterization of a nanomaterial span from particle size, shape, surface areas, chemical configurations and ultimately physico-mechanical aspects like those of hardness, softness, brittleness, ductility, porosity etc.

The techniques available for characterization of nanomaterials are extremely diverse and are applied accordingly on the basis of properties and sources of the nanomaterials to be examined or analyzed. For example, the analysis of airborne nanomaterials requires far lesser preparation as compared to those from the soil where we have to deal with a great deal of heterogeneous surface treatments. In case of source being the soil, we have to go for elaborate procedures for isolation of nanoparticles from the mixtures containing many other materials of different morphologies. Another major factor affecting the characterization of natural nanomaterials is the nature of information required. For instance, Scanning Electron Microscopy (SEM) and Atomic Force Microscopy (AFM), which give information about the surface configurations, require very different sample preparation when compared to Transmission Electron Microscopy (TEM), which fetches the details of internal structure of a material. Sometimes the nanoparticles are complexed with materials that are either natural or anthropogenic in background, such that it becomes difficult to isolate them. In such cases, the characterization of the nanomaterials can't be presumed to be carried out properly. The most challenging situation is encountered while dealing with engineered nanomaterials because for them the analysis and characterization procedures are highly sophisticated and necessitate the analysis of single particles.

The chief methods available for isolation of nanoparticle fractions from the different liquid media include size exclusion chromatography, membrane filtration, ultrafiltration, centrifugation and several others with varying principles [77-80]. The first level of characterization of nanomaterials commences with the application of scanning and transmission electron microscopy techniques which give us vital information about the surface morphology, size of the nanoparticles and the chemical composition of mounted samples in a vacuum environment. The estimation of particle size in liquid mediums via online modules can be obtained by the technique of Dynamic Light Scattering (DLS) [81]. In addition, there is another module of spectroscopy known by the name Inductive Coupled Mass Spectrometry (ICP-MS); which is a very efficient means for obtaining the corresponding chemical information [82]. For the determination of extremely crude nanomaterials as single particles, another spectroscopy technique is popularly used that is known by the name Single-Particle Laser Microprobe Mass Spectrometry (LAMMS) which can provide critical chemical composition information about single particles isolated from collected fractions [83].

$\mathrm{X}$-ray absorption spectroscopy is another module that gives us vital information about the biotransformation of charged nanoparticle species those is present in combination with other materials. This method tells about the change in oxidation state of the nanoparticles as a result of their increased biotransformation tendencies and the corresponding composition in the vicinity of a particular analyte. Studies using this technique have proved the existence of cerium oxide nanoparticles in the soybean tissues with no biotransformation [84].

\section{Biochemical Fate of Nanoparticles in the Natural Environments}

In addition to the impact of various nanomaterials within the domains of biodiversity on the earth, the final phenomenon of biodegradation, bioaccumulation and biological persistence must also be given a sound consideration. These are the natural processes that happen to every material that has been discarded. Now how these events shape up for nanomaterials is really interesting. The reason for this auspicious interest in nanomaterials is obviously due to their unusual material properties which not only makes these materials small but a special kind of small. Biodegradation and bioaccumulation are some natural characteristics of a material. These are therefore highly dependent on physical and chemical properties of the material concerned, its structural features and mode of synthesis as this has a mark on the final complexity of material, in case of nanomaterials. The text ahead mentions some key biodegradation and bioaccumulation routes associated with those of nanomaterials.

\section{Biodegradation of nanomaterials}

Biodegradation can be simply defined as the phenomenon by which a material gets degraded through biological agencies, after it has been discarded as waste in the environment. This is a completely natural activity and involves some unique microbial actions which result in the conversion of a discarded material into an environmentally recyclable form. This is performed mostly by parasites and saprophytes, which are therefore labeled as natural scavengers of the biosphere. If a material is not biodegradable it has a tendency to persist in the environment and get manifested as a toxic material, either by entering the food chains via plants or animals or by mixing with other wastes in the environment and getting chemically complex in their nature.

When nanomaterials or nanoparticles discarded as waste are biodegraded, they get broken down into still smaller forms; resulting in significant changes in their chemical and physical properties as well as their surface characteristics. Of late, with the realization of toxic effects and aftermaths of the nanomaterials, efforts and studies for evaluating the biodegradation of the nanomaterials have received a considerable attention. A large number of nanomaterials, currently in use, are composed of inherently non-biodegradable materials. This is chiefly because of the complex chemical composition of these materials that includes inorganic metals, their oxides, ceramics and their further complex analogs which in native forms do not undergo biodegradation easily. Interesting studies based on the degradation of fullerenes have suggested that after a period of twelve hours, fullerenes are metabolized by wood decaying fungi, hinting that fullerenes are biodegradable [85]. Otherwise with the ever increasing threats to the environment, the biodegradability of nanomaterials has become a serious concern and has a strong bearing on their properties and chemical backgrounds. There is a key role of the nanomaterial toxicity and biodegradability in the wastewater treatment strategies also. Often, nanoparticles of transition metals are employed for waste water treatment since they have good catalytic properties. Unfortunately, these nanoparticles form aggregates or lumps and exert toxic effects on the degrading microorganisms in the waste water stream including those of bacteria and fungi. Studies with fullerenes creating this sort of disturbance, in the form of colloidal structures during the aerobic and anaerobic treatment of waste waters, have been reported $[86,87]$.

\section{Bioaccumulation and persistence of nanomaterials}

There is an increasing tendency of nanomaterials to exist and 
persist in nature. This is largely due to their unique physicochemical properties, the absence of gravitational influences in nanomaterials and their unpredictable and sometimes farfetched interactions with other materials in the nature. Nanoparticles of different background have been observed to get bio-accumulated and magnified in food chains through the biotic components of an ecosphere such as bacteria, fungi, humans and even in the plants through the absorption by several routes [88]. The tendency of settling and reaching saturated inertness is almost absent in nanomaterials as they are extremely small in size and are free from any gravitational controls. Sometimes nanoparticles attain more complex configurations by combining with numerous other micro and macro scaled particles. The reason behind toxic effects is the existence of only these combined forms of the nanomaterials, which are highly unstable and keep on participating in different chemical reactions in one way or the other till they finally attain a stable minimum energy configuration. Moreover, the change in environmental parameters further complicates the toxicities of these reactive nano-complexed materials. The influence of temperature, $\mathrm{pH}$ and pressure on the behavior of these heterogeneous nanostructures is very delicate and makes them potent toxicological customers. This has been observed very evidently in case of iron nanoparticles which change their oxidation states by engaging in enzymatic redox reactions that eventually result in changing the oxidation states, chemical properties and ultimately the interaction behavior of the metal [89]. Biological persistence of a material in nature can be understood in terms of its inherent ability to exist in the nature for indefinitely longer durations, either in native or in combined forms. This is very critically dependent on the natural phenomenon of biodegradability. More is the biodegradability of a material, lesser will be its persistence and vice-versa. The influence of photochemical factors on the complexed metallic nanoparticles also has a significant impact on their integrated biological activities as the photochemical impact could have a strong bearing on their structure-dependent functional attributes.

\section{Risk Assessment Strategies for Nanotoxicology}

Risk assessment of nanomaterials broadly deals with the estimation of the potential threats of the nanomaterials to the environment as well as the diverse living organisms spanning the life on earth. With the rapid and almost blind race in terms of scientific and technological interventions in different walks of life, the exposure to nanomaterials has multiplied and has reached almost an unavoidable stage. The type of nanomaterials responsible for toxicity manifestation can vary from natural to man-made and even to the engineered morphology. It is surprising to recall here that all the nanomaterials are not harmful and induce the toxic effects. Some nanomaterials have been in use since ancient times like those of carbon black and titanium dioxide and possess low toxicities. The fact that smaller a material, the greater is its toxicity is not always true and needs a fresh consideration. In this sense, we can say that nanomaterials are twisting in their biophysical and physicochemical attributes in fluctuating manners, which are sometimes harmful depending on their usage and concentration while at other times; they do not possess any major risk. So far as proper and detailed reliable risk assessment information on the nanomaterials is concerned, there is no well documented literature being reported till date, thereby leading to the speculations of several misconceptions roaming around in the scientific minds. For nanomaterials to pose a risk there must be a potential for both exposure and a hazard, that in conventional terms is known by the name toxicity. Exposure to nanomaterials varies in accord with the corresponding source, which can vary from a research laboratory to a hydrocarbon industry, petrochemical industry and semi-conductor engineering companies and so on. Other than the source, the parameters affecting the exposure to the nanomaterials include their transporting carriers via different natural agencies, their persistence in the environment and the further existence of segregation and entanglement. Most of the studies conducted to ascertain the exposure to nanomaterials with respect to an assessment of their consequent toxicities, have focused on their aerial transport. The studies on direct interactions via dermal exposure or by active transport are far too limited in number $[90,91]$ In a similar manner, the hazards of nanomaterials have also been analyzed to a very local aspect; majorly highlighting on their possible transport mechanisms through lungs and related respiratory organs. The primitive work regarding the toxicities of nanomaterials has dealt with their possible risks while being used for cancer treatment and drug delivery applications in the biomedical domain. Very recently, the focus of interest has shifted to study the same in bacteria and aquatic organisms. The use of certain specialized nanostructures like fullerenes and quantum dots for highly diverse applications, ranging from drug delivery to accurate damaged tissue diagnosis has also been seen as a significant breakthrough. Here engineered nanoparticles are preferred as their surface needs to be tagged with the hydrophilic groups to make them compatible with the drug molecules and also with the body systems. Besides biomedical domain, a number of advanced industrial operations and mechanisms, involving the functionalization of carbon nanotubes, the incorporation of fullerenes is a profuse dimension for complication of nanoscale hazards. The facts like for how long these nanostructures will persist within the human bodies, how will they be cleared out of the body, the possible altered cellular responses they may lead to, need to be properly and logically answered if we are to know about the toxic responses generating from these interactions. Some nanomaterials become toxic by means of aggregation and coalescence while others become beneficial for the intended use in the living systems. For instance, carbon based nanostructures when get aggregated are tougher to clear the respiratory barriers. On the other hand, fullerenes, show higher solubility and improved antibacterial properties when aggregated in aqueous environments [92].The unprecedented use of nanomaterials which have undergone exhaustive surface modifications, remains one of the most challenging feature to be analyzed. Thus, determining the nature and specific details of cellular and environmental response associated with a particular nanomaterial remains the first and most important step in assessing potential risks, on the basis of which, design and usability prospects can be modulated.

\section{Parameters and focus of risk assessment}

Health and safety issues regarding the use of nanomaterials in several applications have become a very serious issue of concern until recently. The first issue of susceptible toxicity was reported with a German product known as Magic Nano Spray [93]. Fortunately, further investigation revealed no active participation of the nanomaterial in the hazard concerned. Nevertheless, the hype of the topic created some unnecessary and illogical misconceptions. Moreover, with majority of technological aspects being still in the infancy stage and the public perception not being so strong and supportive, the commercial acceptance of the nanotechnology in devices has witnessed a widespread reluctance. In some countries, due to little knowledge about the possible pros and cons, environmental conservation organizations and related movements (stimulated by political influences) have been even forcing for a complete ban on the use of nanomaterials. To address the mounting trouble, US Environmental Protection Agency (EPA) has suggested some risk mitigating strategies for the possible control and arrest of uncertain nanotoxicological issues. The very basic issues that have been assumed as the backbone or platform for the design 
Identification of Nanomaterial Toxicology
Exposure Assessment: Particle behavior, routes of entry use overall product
Hazard Assessment: Chemical composition, particle size, structure, shape and properties

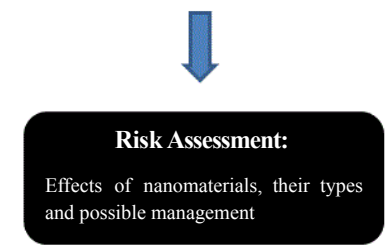

Figure 3: An outline of the nanotechnological risk assessment.

of ethical measures have been laid on the basis of uncertainty in the behavior. The proposed suggestions by EPA include the analysis based on probabilistic modeling and predicting the possible structure-activity relationships through (QSAR).

The key to develop a strange hold tap on the adverse effects of nanomaterials is to have a sound knowledge about their possible physical and chemical behaviors in the nature. This has been a very tough issue for consideration because there are a number of factors which decide about the particular kind of response of a nanomaterial at a particular concentration, in a particular environment and on particular parameters of environment. Now, when we come to analyze these issues with respect to the nanomaterials, we find that which factors need to be taken as more important and which others could be negotiated with, is a tough and risky choice. That is why models proposed by EPA during its latest suggestions were found true only with respect to the conventional large scale materials and are not feasible to explain the behavior of nanomaterials [94]. The International Risk Governance Council (IRGC) has given a detailed set of guidelines for the global risk governance of nanotechnology.

It has also postulated some guidelines for the drafting the experimental models in context with the prediction of behavior of different nanomaterials with reference to analysis of their potential toxicities (Figure 3).

\section{Precise and More Effective Sensing Strategies for Nanomaterials: Current Advances}

Having gained such a deep insight regarding the likelihood mechanisms which can peculiarly render a nanomaterial deleterious from the point of view of healthcare and environmental persistence, the platform is very rife to know about the characteristics shaping up the corresponding toxic responses of nanocarriers in the particular domain of drug delivery. Interestingly, most of the nanocarriers employed or engaged for the application of drug delivery carry their active ingredients in dispersed form or in associations which could favor stimuli triggered targeted release. It is the medium through which nanoparticles exert their characteristic influences, in terms of their molecular motions which are, in turn, specifically dictated by their unique and characteristic interactions and mutual energy exchanges.
Therefore, it is highly logical to study the interaction properties of dispersed nanoparticles, to structurally track and elucidate their medium-interface imposed and contributing behaviors.

In this reference, literature is enriched with very interesting contributions predicting the rationality of physicochemical characterization pertaining to the determination of nanomaterial toxicity [27,95-99]. Almost all of these worthy literature contributions pinpoint to some major aspects, ranging from size, shape, aspect ratio, surface area and finally to surface functionalization. Figure 4 gives a glimpse of all these vital fundamental aspects along with the corresponding predicted aspects these can lead to. However, the turning point of the overall story, remains the fact that most of the conventional physicochemical properties in case of engineered or the so called presumably risk prone nanomaterials dictate the element of unpredictability without actual contact with the living cells. This leaves us with an equally poised task to study the interaction of nanocarriers with living organisms under varying conditions. Several research attempts have used nanoparticle encapsulation, nanoparticle conjugation and nanocarriers in the form of nanoemulsions, nanosuspensions, nano liposomes to deliver the drug of interest to the desired location with an objective to maximize its concrete actionmechanism driven pharmacological ability. The question looming high

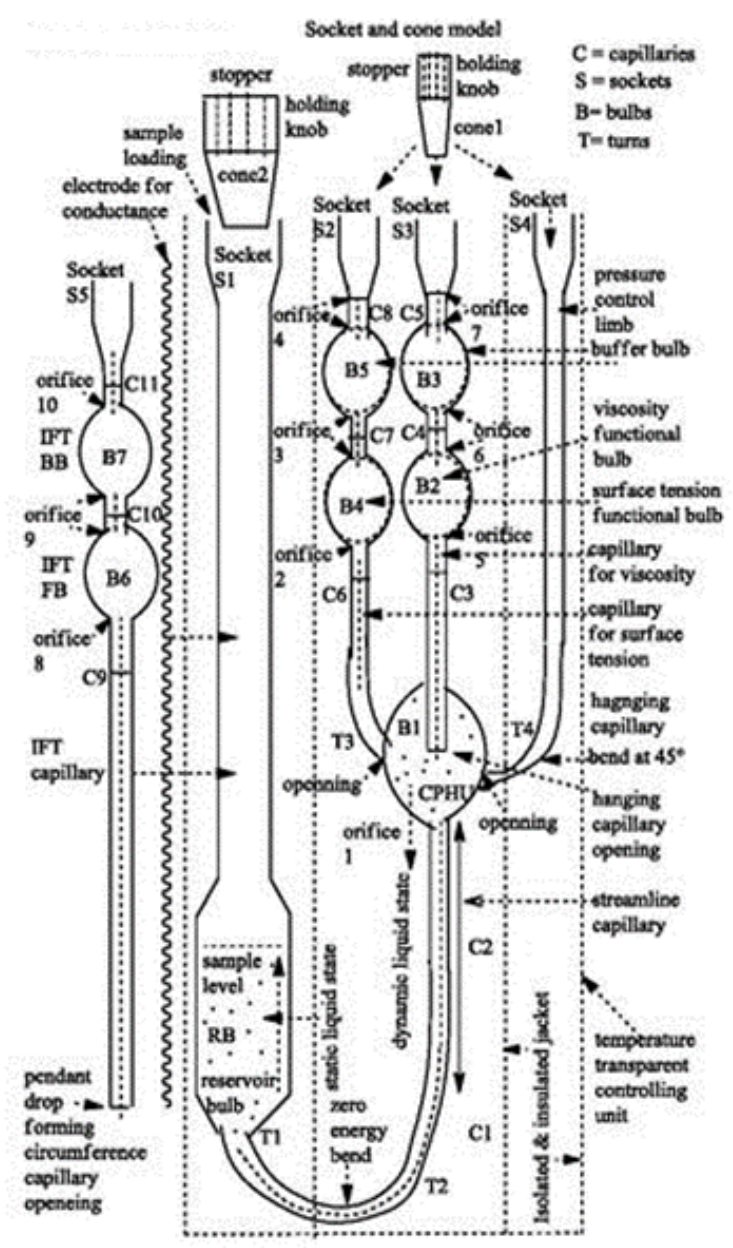

Figure 4: Schematic view of a typical Survismeter instrument. 
is the fact that how the very basic and preliminary liquid based physical properties of surface tension and viscosity are affected from the point of view of a healthy and fit physiological environment, when these unconventional or rather functionalized nanomaterials exert their characteristic activities in due course of time under the physiological and thermodynamic barriers.

It would be of immense interest if we create the living conditions (corresponding to temperature, $\mathrm{pH}$ and cellular environment) and compare these physicochemical properties after the nanomaterials interact with the cells under this created environment. The presumption behind this is that nanomaterials, because of their engineered morphology, undergo numerous fluidic interactions under the physiological constraints and get aggregated. Under these conditions, it is of further interest as upto how long these nanoscale assemblies persist within the bodies of living organisms. Interestingly, here the most important aspect of studying the interactions of these nanomaterials remains the consideration of the possibility that if the energy of these presumed combinations is decreased, the stability of these complexes would be maximized, thereby making them persist for further longer durations and complicating their elimination from living systems. Studies have already focused, repeatedly over the fact that the persistence of engineered nanomaterials within the body results in the problems of oxidative stress and related inflammatory responses and many more complications of different nature.

Under such a scenario, it has become immensely urgent to correlate the dependence of proposed toxicities with the concrete alterations on the highly localized specific and sensitive fundamental physicochemical riders of liquid mixtures, which are almost concurrently studied through the surface tension and viscosity. With respect to these interesting presumptions, a novel invention from Indian soil proves to be a remarkable breakthrough from the point of view of toxicology study through entirely green chemistry based approach. This invention has been coined the name as Survismeter, by the inventor. The instrument has a two-tier provision for the measurement of surface tension and viscosity and it also measures the interfacial tension of solutions in context of specific local chemical environments [98101]. On the basis of these primary parameters, a whole range of essential secondary physicochemical parameters including those of binding energies, wetting coefficients, surface excess concentration, friccohesity and tentropy can be ascertained; thereby conveying vital information about the probable effects, the nanomaterial dispersions can lead to. This invention serves as the backbone for the assessment of interactions of different materials with various solvents and at different temperatures. The immensely beneficial task remains the fact that through the estimation of physicochemical parameters, one can estimate the medium which would be suitable for the proper distribution of pharmaceutical ingredients of the engineered medicinal formulations.

The technical aspects and the fundamental working mechanism has been discussed in a comprehensive manner by the inventor himself through a compilation of 2006, reported in the Journal of Biophysical and Biochemical Methods. This compilation discusses the functional aspects of Survismeter working with respect to the measurement of surface tension and viscosity [98].

At once, one of these parameters can be measured. Figure 4 represents the typical model of a Borosil Mansingh Survismeter (BMS). To begin with, the sample is injected into reservoir bulb section till it is two-third filled. Thereafter, the pressure and the viscosity valves are closed with respective stoppers and the sample is sucked up through the surface tension probe section.

Similarly, for the measurement of viscosity $(\eta)$, the surface tension $(\gamma)$ and the pressure valve sections are kept closed and liquid to be tested is sucked through the viscosity limb. Care should be taken so as to avoid the abrupt formation of bubbles or effervescence in either of the probes while sample is being sucked off through a sucker. This is more important in case the liquid being tested is enriched or attenuated or functionalized for a specific task through lacing with a surfactant or any other chemical stimulant. The two properties are measured through the respective measurement of Pendant Drops (PDN) and Viscous Flow Time (VFT).

For measurement, firstly, the calibration with the blank, which is preferably the major solvent component of the formulation to be tested, is done, with respect to which the droplet number $\left(\mathrm{n}_{\mathrm{o}}\right)$ and viscous flow time $\left(\mathrm{t}_{\mathrm{o}}\right)$ were obtained. These parameters are determined over a specific temperature, using a standard counter and the stop-watch. The most interesting aspect of this instrument is that the measurements corresponding to pendant drop count and the viscous flow time are highly reproducible. The surface tensions $(\gamma)$ and viscosities $(\eta)$ are thereafter calculated using the following formulae:

$$
\begin{aligned}
& \gamma=\left[\left(\mathrm{n}_{\mathrm{o}} / \mathrm{n}\right)\left(\rho / \rho_{\mathrm{o}}\right)\right] \gamma_{\mathrm{o}} \\
& \eta=\left[\left(\mathrm{t} / \mathrm{t}_{\mathrm{o}}\right)\left(\rho / \rho_{\mathrm{o}}\right)\right] \eta_{\mathrm{o}},
\end{aligned}
$$

where, $\rho$ and $\rho$ o denote the temperature specific densities of tested and reference liquid at $\mathrm{a}, \mathrm{n}$ stands for the droplet number of sample to be investigated, $\eta_{0}$ and $\gamma_{0}$ denote the viscosity and surface tension of the reference solvent.

For accuracy and minimization of error in measurement, three readings were taken corresponding to each measurement and the resulting concordant reading was taken as final value. The maintenance of temperature constancy was achieved via keeping the overall setup in a glass chamber filled with Millipore water and connected with a water bath cum temperature control probe. The detailed methodology and operational fundamentals have been put to the forefront by the inventor himself in a very brief but highly informative research article, of 2007. This literature source further sheds more intense knowledge with respect to the characterization of fluid streams carrying environmental pollutants. This is reflected through the postulation of interfacial tension, by which we can study the consequence of reactivity of nanomaterials in a waste stream carrying nanomaterials in suspended form as waste [102,103]. Figure 5 further depicts some more informative snapshots of this cutting edge innovation from Indian soil.

Till date, numerous advanced research investigations have been reported through the application of this instrument and those too, in the diverse fields of organometallic chemistry, formulation validation, interaction chemistry and several others [104-110]. Since the instrument has a manual approach, it therefore leaves a very little scope for human error to alter or interfere with the corresponding measurement and therefore, presents a highly well-suited technique for analysis at the nanoscale.

Interestingly, the fundamental science of surface tension in liquids also explains the extent of dispersion of a solute in a liquid formulation and its relative ease of availability over and around the surface.

Some interesting scientific reports prevail in literature, which discuss about the importance of surface tension maintenance of the blood in a normal human being [111]. These aspects also discuss the role of temperature and its critical influence in the alteration 

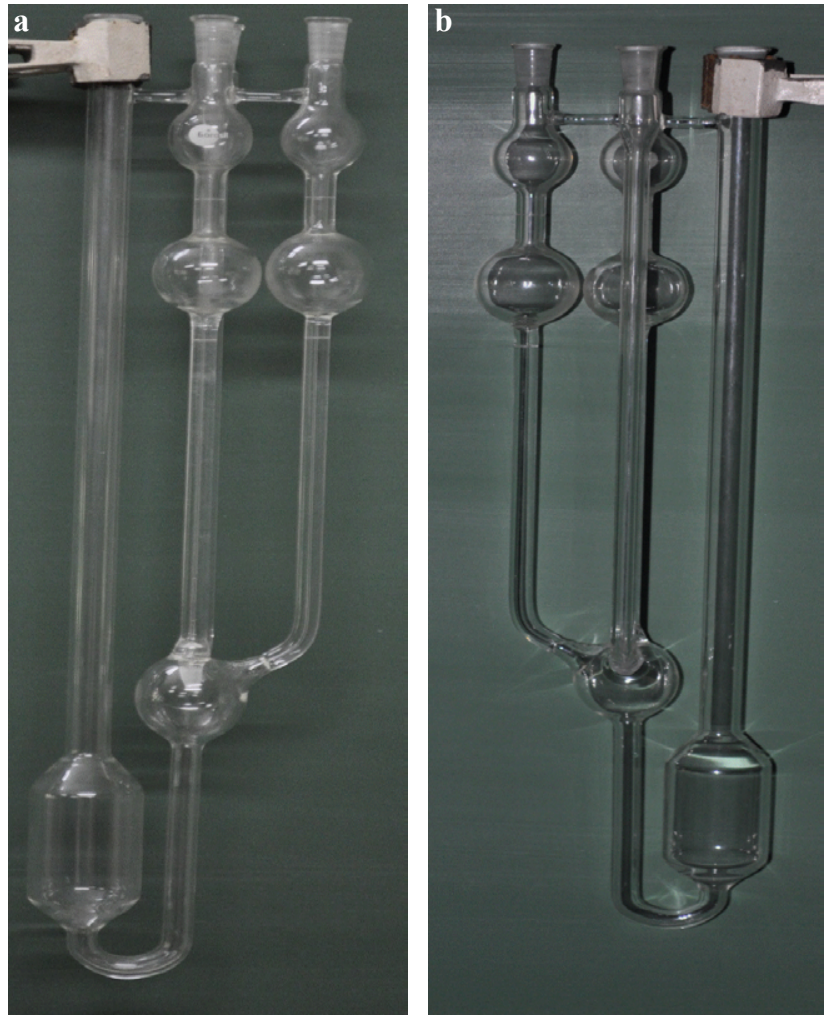

Figure 5: A closer glimpse of cross-sections of Borosil Mansingh Survismeter.

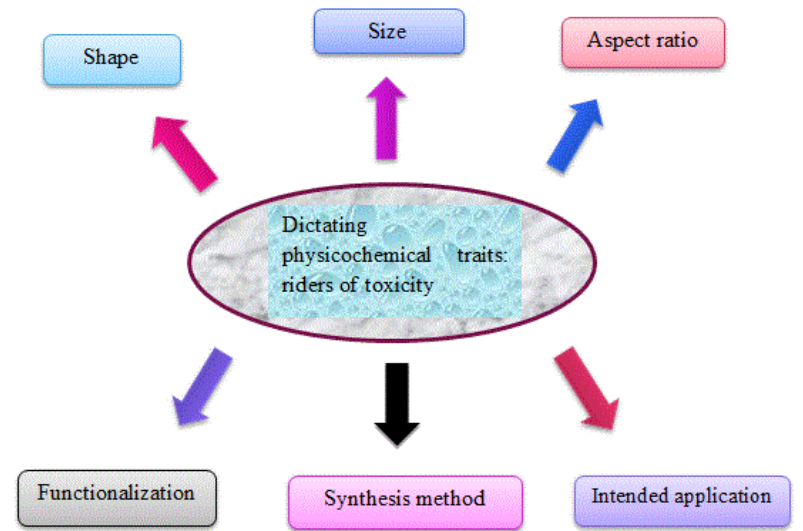

Figure 6: The decisive toxicity physicochemical and fundamental traits of nanoparticles.

and functioning as a probable source of thermodynamic impact. Interestingly enough, if we determine the surface tension of the nanocarriers delivered drug with the blood, it will give a quantitative description of the drug interaction within the biological systems, through which we can ascertain corresponding consequences under the physiological conditions (Figure 6). Further, if we are also able to stabilize the temperature in equivalence to that of the body temperature, it will enable us to know about the possible effect of engineered drug formulation on our body tissues, with respect to the limits of surface tension a normal human body can bear. Subsequently, the specified deleterious role of each structural component can be deduced. The advanced applications of altered drug delivery of numerous drugs possessing high therapeutic potential but having low retention in the native form can be very actively optimized through the use of this novel innovation $[103,105]$.

This invention also facilitates the measurement of the wetting coefficient of low or poorly water soluble compounds, which is the backbone for determining the material interactions at the nanoscale, in terms of exclusive mutualistic variations of cohesive and intermolecular forces. The extent to which a liquid interacts with a solid on a characteristic surface is determined by this property of wetting coefficient, the phenomenon or patterns like why a liquid always tends to form a spherical shape on the surface of a solid, or as to what could be its possible implication if we alter the wetting nature to an extent that gives rise to a shape other than that of spherical. The dissolution of drugs is being increasingly modulated and attenuated to mend their low therapeutic activities, and, in such cases the characteristic effect of a particular carrier can be assayed through calculation and measurement of wetting coefficient. Further, in this particular dimension, the use of nanocarriers like those of engineered nanoparticles, dissolution through nanoscale formulations etc. can be excellently studied to determine their biological feasibility through analysis of physicochemical properties of surface tension, viscosity, interacting abilities, friccohesity determination and several others.

\section{Chemical potential of engineered nanomaterials: Friccohesity influence}

The interactions of nanomaterials with the living cells present some very intense and intriguing correlations with the prospects to know about their possible threats and toxicological risks. This is with reference to the fact that upon being delivered to the body through a specific carrier, the carrier undergoes surveillance within the periphery of so many distinct tissues, each of which has a specific optimum chemical and biological environment. Under these rigid and specific situations, the interactions of nanomaterials with cellular components are critically dictated by the breaking of cohesive forces and the development of intermolecular forces. This tendency, measured by the direct parameter of Friccohesity, gives us the knowledge about the extent of cohesive force destruction and the corresponding intermolecular force conversion. Friccohesity is a unique molecular force transition rider, through which the dictations of different types of forces enable us to determine the chemical behavior of a particular material. It signifies the scientific unveiling of a novel science in it, as it gives the quantitative idea of the formation of newer entities from the very basic idea of chemical bond formation. Its relevance in terms of toxicity prediction seems to be highly important since invoking of toxicity requires interactions with the body system. As a further continuance of this constraint, the inherent structural bonding of the nanomaterial in use, mediated via cohesive forces, is gradually replaced by intermolecular forces. Numerous interesting studies have been explained through the proposition of friccohesity at the molecular level, encompassing from drug interactions, deduction of structure activity relationships, the thermodynamically supported interaction patterns of amino acids with many chelating atoms and henceforth $[103,104]$. The relative extent of intermolecular force development determines the quantitative aspect of probabilistic toxic cellular responses that a particular nanomaterial can invoke from within the body system. We can say that friccohesity is a molecular level operator linking cohesive forces and intermolecular forces, both of which are 
inversely proportional to each other at one particular instant of time. This novel operator can help to deduce the presence of definite force content with the corresponding proportion of the generated risk. This is because the bond formation and destruction is very evidently, the backbone of molecular interactions at the physical, chemical and biological, biophysical ad biochemical level.

Application with respect to dissolution studies: Nanoemulsions: The dispersion of diverse kinds of materials, ranging from nutrients, growth factors, hormones and a number of other important precursors is an integral characteristic of all the living organisms. This feature leads to the formation of emulsions and sometimes, the solute is present in very minute extents, thereby leading to the genesis of nanoemulsions. The proposed innovation of Survismeter is a pioneering weapon to study and characterize these emulsion systems and deduce the deviations of the fundamental physicochemical properties when the nanomaterials are present and are creating the undue influences [107]. The colloidal emulsions play some deeply crucial and unique roles to mediate the biological sanctity of living tissues and functioning. If we have a careful glance at the structure of biomolecules, drugs, nutraceuticals and other important biological entities required by living organism for survival, it is very obvious that these possess functional groups, charge density, $d$-orbital $\pi$-electron localization, charge distribution, alkyl chains of variable lengths and many more interesting structural fluctuations. Each of these physicochemical attribute is befitted with an inherent ability to impart specified interaction abilities and since most of biomolecules are organic in nature, so they are the initiators or originators of intramolecular entropies that have been termed as tentropy, in the proposition of this novel idea. In these conditions, understanding these interactions with the help of structural modalities is very essential. Emulsions are indeed very promising in this regard, particularly because these carry several ingredients and each of these has a separate distinct and uniquely defined chemical potential, due to which they develop distinct Localized and Global Equilibrium Thermodynamic (LET and GET) constraints. If a stable, specific and critically optimum combination of all such constituents is pursued, it will lead to the establishment of the synergistic effect of the individual components. Friccohesity attempts to explain the concepts of drug binding and solvent dissolution and synergism in a very critical manner, due to which it is feasible to combine together the chemical potential of two distinct phases into the regulation of their inherent energy capacities. A characteristic chemical potential is thereby generated, out of the controlled interactions, which is purely through the natural basis and attempts to form stable dispersions. This chemical potential is altered in the cases where nanomaterials tend to lend a toxic effect and negatively interfering role with respect to growth and development in living organisms.

\section{Elucidation of Prevailing Physiological Interactions: Tentropy Insight}

Threats to living organisms at a localized level are dependent on a number of closely interlinked intrinsic factors; the proper understanding of which can be made only through a detailed knowledge of the probable interactions that have significant likelihood of being prevalent. In this context, if we carefully analyze the bodily interactions with any foreign material, one point of consensus being arrived at is the fact that living body is majorly composed of carbon. Further, it is evident that carbon is tetravalent in nature and thus has a scope for binding different kinds of materials, which is possible through its ability to undergo significant level of catenation. By virtue of its rapid ease to form multiple bonds with different substances, the carbon based nanomaterials and engineered nanostructures pose a significant risk of getting entangled within the biomolecular structures inside the living organisms. These mechanistic and specific interaction based biochemical responses are the chief sources of oxidative and inflammatory stress based disorders and complications. Combination of these carbon based structures with each other leads to the development of hydrophobic forces and constant interconversion of cohesive forces into intermolecular forces till the attainment of a particular configuration, having a definite proportion of each of these forces, with the maximum stability potential is attained. The resultant structural optimizations or formulations of molecules, formed as a result of these interactions have their major activities defined by the probabilistic interactions between the hydrophobic alkyl chain parts and the corresponding foreign substances, which in the present context are the nanomaterials being exposed to.

Almost on the similar lines, the diverse organically driven interaction of a material within a specific environment is specifically defined in terms of a molecular activity operator of tentropy. The tentropy is basically entropy manifested through the possession of tentacles, i.e. in a typical scenario; where organic components, as present mostly within the physiological environments, interact with so many other materials and the presence of hydrophobic long chains in their structures contribute to the gradual complexity of overall interactions developed. The justification of the existence of this physicochemical characteristic of organic entities has been pointed out and discussed at length, by Singh et al. [110], who have wonderfully correlated the existence of molecular force modulation with the existence of these organically mediated interactions. In their contribution, the authors have considered the interactions of Polyvinylpyrrolidone (PVP), carrying polymeric chains with amino acid glycine. The authors have supported the prevalence of tentropy from within the intramolecular level developed feature of entropy [109]. In one other interesting compilation, tentropy has been defined as entropy prevailing within a molecule, or indirectly being termed as intramolecular entropy [112]. Extending the similar concept to the dissolution parameter evaluation, the gradient distribution of molecular forces in fluids, leading to the formation of solutions, mixtures and emulsions through the formation of concurrent molecular forces of variable strengths, has been convincingly discussed. All these literature compilations collectively point out that in any chemical reaction, the reactant species exert some specific frictional influences over each other, in course of which characteristic interaction patterns are generated, some of which develop hydrophobic domains, while the others give rise to entanglements and still others give rise to weakly linked molecularly sensitive associations.

\section{Conclusion}

The emerging threat of nanotoxicology, can, thus be understood as a very unconventional and hazardous and a crucial ethical concern of this technology. Materials form the backbone of every conventional scientific domain and the urge for the development of stronger, faster and comparatively more readily tunable ones is fast emerging as the cornerstone of the interdisciplinary boundaries of nanotechnology. Thus far, the emergence of toxicological risks from the rapid surges in nanomaterial development proves to be a very intriguing issue affecting the sustainable development of the environmental resources. The multidisciplinary design approaches such as those of Quantitative Structural Analysis and Relationship (QSAR) and those of predictive model based computational tools seem to be very vital combative measures to confront this secretly growing nanotechnological menace. In this context, it is equally important to have a sound awareness 
Citation: Malik P, Mukherjee TK, Singh M (2014) Biomedical Nanotoxicology and Concerns with Environment: A Prospective Approach for Merger with Green Chemistry Enabled Physicochemical Characterization. J Microb Biochem Technol S9: 001. doi:10.4172/1948-5948.S9-001

program since the industries dealing with active manufacturing usage of nanomaterials are mostly unaware of the risks of used nanomaterials. An aid to this could be synergized through the development of a comprehensive basic sciences program, vitalized and envisaged through the core characteristics of nanoscale threats. Furthermore, it is not inherently the nanomaterials which lead to this problem whereas, the problem of functionalization in form of sophisticated coatings and functionalization procedures resulting in persistence of otherwise degradable materials seem to be the most critical factor in the aggravating the toxicological attributes of nanomaterials. Another noteworthy aspect in this direction is the dependence of material behavior on the physicochemical properties, since these are the frontline riders of the varying material behavior in different environments. This further necessitates the rationale of risk assessment of the material usability in order to have a tap on their toxicity attributes. The concepts of tentropy and friccohesity thus argue very well to ascertain the material behavior under different chemical vicinities and open the field wide open for the understanding of material behavior in different configurations and optimizations.

\section{References}

1. Buzea C, Pacheco II, Robbie K (2007) Nanomaterials and nanoparticles: sources and toxicity. Biointerphases 2: MR17-71.

2. Mahmoudi M, Hofmann H, Rothen-Rutishauser B, Petri-Fink A (2012) Assessing the in vitro and in vivo toxicity of superparamagnetic iron oxide nanoparticles. Chem Rev 112: 2323-2338.

3. Colvin VL (2003) The potential environmental impact of engineered nanomaterials. Nat Biotechnol 21: 1166-1170.

4. Savolainen K, Alenius H, Norppa H, Pylkkänen L, Tuomi T, et al. (2010) Risk assessment of engineered nanomaterials and nanotechnologies--a review. Toxicology 269: 92-104.

5. Casals E, Vazquez-Campos S, Bastus NG, Puntes V (2008) Distribution and potential toxicity of engineered inorganic nanoparticles and carbon nanostructures in biological systems. Trends Anal Chem 27: 672-683.

6. Oberdörstera E, Zhuc S, Blickley TM, McClellan-Green P, Haasch ML (2006) Ecotoxicology of carbon-based engineered nanoparticles: Effects of fullerene (C-60) on aquatic organisms. Carbon 44:1112-1120.

7. Magrez A, Kasas S, Salicio V, Pasquier N, Seo JW, et al. (2006) Cellular toxicity of carbon-based nanomaterials. Nano Lett 6: 1121-1125.

8. Bottini M, Bruckner S, Nika K, Bottini N, Bellucci S, et al. (2006) Multi-walled carbon nanotubes induce T lymphocyte apoptosis. Toxicol Lett 160: 121-126.

9. Dreher KL (2004) Health and environmental impact of nanotechnology: toxicological assessment of manufactured nanoparticles. Toxicol Sci 77: 3-5.

10. Swiss Report Reinsurance Company 2004.

11. UK Royal Society (2004).

12. European Nano Safe Report (2004)

13. Smijs TG, Bouwstra JA (2010) Focus on skin as a possible port of entry for solid nanoparticles and the toxicological impact. J Biomed Nanotechnol 6: 469-484.

14. Arayachukeat S, Wanichwecharungruang SP, Tree-Udom T (2011) Retiny acetate-loaded nanoparticles: dermal penetration and release of the retinyl acetate. Int J Pharm 404: 281-288.

15. Yah CS, Simate GS, lyuke SE (2012) Nanoparticles toxicity and their routes of exposures. Pak J Pharm Sci 25: 477-491.

16. O'Dowd CD, Jimenez JL, Bahreini R, Flagan RC, Seinfeld JH, et al. (2002) Marine aerosol formation from biogenic iodine emissions. Nature 417: 632-636.

17. Sellegri K, Villani P, Picard D, Dupuy R, O'Dowd C, Laj P (2008) Role of the volatile fraction of submicron marine aerosol on its hygroscopic properties. Atmos Res 90: 272-277.

18. O'Dowd CD, Facchini MC, Cavalli F, Ceburnis D, Mircea M, et al. (2004) Biogenically driven organic contribution to marine aerosol. Nature 431: 676680 .
19. Campbell A, Oldham M, Becaria A, Bondy SC, Meacher D, et al. (2005) Particulate matter in polluted air may increase biomarkers of inflammation in mouse brain. Neurotoxicology 26: 133-140.

20. Oberdorster G, Gelein RM, Ferin J, Weiss B (1995) Association of particulate air pollution and acute mortality: involvement of ultrafine particles? Inhal Toxicol 7: 111-124.

21. Fang J, Yu BY (2013) [Transport behaviors of metal oxide nanomaterials in various soils]. Huan Jing Ke Xue 34: 4050-4057.

22. Ben-Moshe T, Dror I, Berkowitz B (2010) Transport of metal oxide nanoparticles in saturated porous media. Chemosphere 81: 387-393.

23. Kretzschmar R, Sticher H (1997) Transport of humic-coated iron oxide colloids in a sandy soil: Influence of $\mathrm{Ca} 2+$ and trace metals. Environ Sci Technol 31 : 3497-3504.

24. Schrick B, Hydutsky BW, Blough JL, Mallouk TE (2004) Delivery vehicles for zerovalent metallic nanoparticles in soil and groundwater. Chemistry of Materials 16: 2187-2193.

25. Wiesner MR, Lowry GV, Alvarez P, Dionysiou D, Biswas P (2006) Assessing the risks of manufactured nanomaterials. Environ Sci Technol 40: 4336-4345.

26. Han L, Wang P, Dong S (2012) Progress in graphene-based photoactive nanocomposites as a promising class of photocatalyst. Nanoscale 4: 5814 5825.

27. Dingshan Y, Enoch N, Feng Du, Liming D (2010) Metal-Free carbon nanomaterials become more active than metal catalysts and last longer. J Phys Chem Lett 1: 2165-2173

28. Fabian E, Landsiedel R, Ma-Hock L, Wiench K, Wohlleben W, et al. (2008) Tissue distribution and toxicity of intravenously administered titanium dioxide nanoparticles in rats. Arch Toxicol 82: 151-157.

29. Warheit DB, Sayes CM, Reed KL (2009) Nanoscale and fine zinc oxide particles: can in vitro assays accurately forecast lung hazards following inhalation exposures? Environ Sci Technol 43: 7939-7945.

30. Morales MI, Rico CM, Hernandez-Viezcas JA, Nunez JE, Barrios AC, et al. (2013) Toxicity assessment of cerium oxide nanoparticles in cilantro (Coriandrum sativum L.) plants grown in organic soil. J Agric Food Chem 61 6224-6230.

31. Fahmy B, Cormier SA (2009) Copper oxide nanoparticles induce oxidative stress and cytotoxicity in airway epithelial cells. Toxicol In Vitro 23: 1365-1371.

32. Ahamed M, Siddiqui MA, Akhtar MJ, Ahmad I, Pant AB, et al. (2010) Genotoxic potential of copper oxide nanoparticles in human lung epithelial cells. Biochem Biophys Res Commun 396: 578-583.

33. Tourinho PS, van Gestel CA, Lofts S, Svendsen C Soares AM, et al. (2012) Metal-based nanoparticles in soil: fate, behavior, and effects on soil invertebrates. Environ Toxicol Chem 31: 1679-1692.

34. Bystrzejewska-Piotrowska G, Golimowski J, Urban PL (2009) Nanoparticles: their potential toxicity, waste and environmental management. Waste Manag 29: $2587-2595$

35. Dowding JM, Das S, Kumar A, Dosani T, McCormack R, et al. (2013) Cellular interaction and toxicity depend on physicochemical properties and surface modification of redox-active nanomaterials. ACS Nano 7: 4855-4868.

36. Arora S, Rajwade JM, Paknikar KM (2012) Nanotoxicology and in vitro studies: the need of the hour. Toxicol Appl Pharmacol 258: 151-165.

37. Wu Q, Nouara A, Li Y, Zhang M, Wang W, et al. (2013) Comparison of toxicities from three metal oxide nanoparticles at environmental relevant concentrations in nematode Caenorhabditis elegans. Chemosphere 90: 1123-1131.

38. Valavanidis A, Vlachogianni T (2010) Nanomaterials and nanoparticles in the aquatic environment: Toxicological and ecotoxicological risks. Sci Adv Environ Toxicol Ecotoxicol Issues.

39. Griffitt RJ, Luo J, Gao J, Bonzongo JC, Barber DS (2008) Effects of particle composition and species on toxicity of metallic nanomaterials in aquatic organisms. Environ Toxicol Chem 27: 1972-1978.

40. Artells E, Issartel J, Auffan M, Borschneck D, Thill A, et al. (2013) Exposure to cerium dioxide nanoparticles differently affect swimming performance and survival in two daphnid species. PLoS One 8: e71260.

41. Asghari S, Johari SA, Lee JH, Kim YS, Jeon YB, et al. (2012) Toxicity of 
Citation: Malik P, Mukherjee TK, Singh M (2014) Biomedical Nanotoxicology and Concerns with Environment: A Prospective Approach for Merger with Green Chemistry Enabled Physicochemical Characterization. J Microb Biochem Technol S9: 001. doi:10.4172/1948-5948.S9-001

Page 13 of 14

various silver nanoparticles compared to silver ions in Daphnia magna. $J$ Nanobiotechnology 10: 14

42. Curtis J, Greenberg M, Kester J, Phillips S, Krieger G (2006) Nanotechnology and nanotoxicology: a primer for clinicians. Toxicol Rev 25: 245-260.

43. Hagens WI, Oomen AG, de Jong WH, Cassee FR, Sips AJ (2007) What do we (need to) know about the kinetic properties of nanoparticles in the body? Regul Toxicol Pharmacol 49: 217-229.

44. Oberdörster G, Oberdörster E, Oberdörster J (2005) Nanotoxicology: an emerging discipline evolving from studies of ultrafine particles. Environ Health Perspect 113: 823-839

45. Trop M, Novak M, Rodl S, Hellbom B, Kroell W, et al. (2006) Silver-coated dressing acticoat caused raised liver enzymes and argyria-like symptoms in burn patient. J Trauma 60: 648-652.

46. Desai P, Patlolla RR, Singh M (2010) Interaction of nanoparticles and cellpenetrating peptides with skin for transdermal drug delivery. Mol Membr Biol 27: 247-259

47. Gopee NV, Roberts DW, Webb P, Cozart CR, Siitonen PH, et al. (2007) Migration of intradermally injected quantum dots to sentinel organs in mice. Toxicol Sci 98: 249-257.

48. Kim S, Lim YT, Soltesz EG, De Grand AM, Lee J, et al. (2004) Near-infrared fluorescent type II quantum dots for sentinel lymph node mapping. Nat Biotechnol 22: 93-97.

49. Asgharian B, Price OT (2007) Deposition of ultrafine (nano) particles in the human lung. Inhal Toxicol 19: 1045-1054.

50. Warheit DB, Sayes CM, Frame SR, Reed KL (2010) Pulmonary exposures to Sepiolite nanoclay particulates in rats: resolution following multinucleate giant cell formation. Toxicol Lett 192: 286-293.

51. Song XL, Li B, Xu K, Liu J, Ju W, et al. (2012) Cytotoxicity of water-soluble mPEG-SH-coated silver nanoparticles in HL-7702 cells. Cell Biol Toxicol 28 : 225-237

52. Choi O, Deng KK, Kim NJ, Ross L Jr, Surampalli RY, et al. (2008) The inhibitory effects of silver nanoparticles, silver ions, and silver chloride colloids on microbial growth. Water Res 42: 3066-3074.

53. Blaser SA, Scheringer M, Macleod M, Hungerbühler K (2008) Estimation of cumulative aquatic exposure and risk due to silver: contribution of nanofunctionalized plastics and textiles. Sci Total Environ 390: 396-409.

54. Long TC, Saleh N, Tilton RD, Lowry GV, Veronesi B (2006) Titanium dioxide (P25) produces reactive oxygen species in immortalized brain microglia (BV2): implications for nanoparticle neurotoxicity. Environ Sci Technol 40: 4346-4352.

55. Dunford R, Salinaro A, Cai L, Serpone N, Horikoshi S, et al. (1997) Chemical oxidation and DNA damage catalysed by inorganic sunscreen ingredients. FEBS Lett 418: 87-90.

56. Vevers WF, Jha AN (2008) Genotoxic and cytotoxic potential of titanium dioxide (TiO2) nanoparticles on fish cells in vitro. Ecotoxicology 17: 410-420.

57. Park KH, Chhowalla M, lqbal Z, Sesti F (2003) Single-walled carbon nanotubes are a new class of ion channel blockers. J Biol Chem 278: 50212-50216.

58. Nikula KJ, Snipes MB, Barr EB, Griffith WC, Henderson RF, et al. (1995) Comparative pulmonary toxicities and carcinogenicities of chronically inhaled diesel exhaust and carbon black in F344 rats. Fundam Appl Toxicol 25: 80-94.

59. Driscoll KE, Carter JM, Howard BW, Hassenbein DG, Pepelko W, et al. (1996) Pulmonary inflammatory, chemokine, and mutagenic responses in rats after subchronic inhalation of carbon black. Toxicol Appl Pharmacol 136: 372-380.

60. Handy RD, Henry TB, Scown TM, Johnston BD, Tyler CR (2008) Manufactured nanoparticles: their uptake and effects on fish--a mechanistic analysis. Ecotoxicology 17: 396-409.

61. Hussain SM, Hess KL, Gearhart JM, Geiss KT, Schlager JJ (2005) In vitro toxicity of nanoparticles in BRL 3A rat liver cells. Toxicol In Vitro 19: 975-983.

62. Warheit DB (2001) Inhaled amorphous silica particulates: what do we know about their toxicological profiles? J Environ Pathol Toxicol Oncol 20 Suppl 1: 133-141.

63. Yu T, Malugin A, Ghandehari H (2011) Impact of silica nanoparticle design on cellular toxicity and hemolytic activity. ACS Nano 5: 5717-5728.

64. Kale SN, Arora S, Bhayani KR, Paknikar KM, Jani M, et al. (2006) Cerium doping and stoichiometry control for biomedical use of $\mathrm{La} 0.7 \mathrm{Sr} 0.3 \mathrm{MnO} 3$ nanoparticles: microwave absorption and cytotoxicity study. Nanomed 2: 217-221.

65. Fernández-Urrusuno R, Fattal E, Féger J, Couvreur $P$, Thérond $P$ (1997) Evaluation of hepatic antioxidant systems after intravenous administration of polymeric nanoparticles. Biomaterials 18: 511-517.

66. Ryman-Rasmussen JP, Riviere JE, Monteiro-Riviere NA (2006) Penetration of intact skin by quantum dots with diverse physicochemical properties. Toxicol Sci 91: 159-165.

67. Hardman R (2006) A toxicologic review of quantum dots: toxicity depends on physicochemical and environmental factors. Environ Health Perspect 114: 165172.

68. Li N, Sioutas C, Cho A, Schmitz D, Misra C, et al. (2003) Ultrafine particulate pollutants induce oxidative stress and mitochondrial damage. Environ Health Perspect 111: 455-460.

69. Soto K, Garza KM, Murr LE (2007) Cytotoxic effects of aggregated nanomaterials. Acta Biomater 3: 351-358.

70. MacNee W, Donaldson K (2003) Mechanism of lung injury caused by PM10 and ultrafine particles with special reference to COPD. Eur Respir J Suppl 40 : $47 \mathrm{~s}-51 \mathrm{~s}$

71. Foldbjerg R, Dang DA, Autrup H (2011) Cytotoxicity and genotoxicity of silver nanoparticles in the human lung cancer cell line, A549. Arch Toxicol 85: 743750

72. Kim JS, Adamcakova-Dodd A, O'Shaughnessy PT, Grassian VH, Thorne PS (2011) Effects of copper nanoparticle exposure on host defense in a murine pulmonary infection model. Part Fibre Toxicol 8: 29.

73. Chen X, Schluesener HJ (2008) Nanosilver: a nanoproduct in medical application. Toxicol Lett 176: 1-12.

74. Smith MW, Thomas NW, Jenkins PG, Miller NG, Cremaschi D, et al. (1995) Selective transport of microparticles across Peyer's patch follicle-associated M cells from mice and rats. Exp Physiol 80: 735-743.

75. Powers KW, Brown SC, Krishna VB, Wasdo SC, Moudgil BM, et al. (2006) Research strategies for safety evaluation of nanomaterials. Part VI. Characterization of nanoscale particles for toxicological evaluation. Toxicol Sci 90: 296-303.

76. Rocha JC, de Sene JJ, dos Santos A, Toscano IA, Zara LF (2000) Aquatic humus from an unpolluted Brazilian dark-brown stream: general characterization and size fractionation of bound heavy metals. J Environ Monit 2: 39-44.

77. Alappat CF, Kannan KP, Vasanthi NS (2012) Biosynthesis of Au nanoparticles using the endophytic fungi isolated from Bahunia variegata. IRACSTEngineering Science and Technology: An International Journal 2: 2250-3498.

78. Chen B, Selegue JP (2002) Separation and characterization of single-walled and multiwalled carbon nanotubes by using flow field-flow fractionation. Anal Chem 74: 4774-4780.

79. Biswas P, Wu CY (2005) Nanoparticles and the environment. J Air Waste Manag Assoc 55: 708-746

80. Chen B, Beckett R (2001) Development of SdFFF-ETAAS for characterizing soil and sediment colloids. Analyst 126: 1588-1593.

81. McMurry PH (2000) A review of atmospheric aerosol measurements Atmospheric Environ. 34: 1959-1999.

82. López-Moreno ML, de la Rosa G, Hernández-Viezcas JA, Castillo-Miche $\mathrm{H}$, Botez CE, et al. (2010) Evidence of the differential biotransformation and genotoxicity of $\mathrm{ZnO}$ and $\mathrm{CeO} 2$ nanoparticles on soybean (Glycine max) plants. Environ Sci Technol 44: 7315-7320.

83. Filley TR, Ahn M, Held BW, Blanchette RA (2005) Investigations of fungal mediated (C60-C70) fullerene decomposition. Preprints of extended abstracts presented at the 229th ACS National Meeting, American Chemical Society 45 446-450.

84. Dhawan A, Taurozzi JS, Pandey AK, Shan W, Miller SM, et al. (2006) Stable colloidal dispersions of $\mathrm{C} 60$ fullerenes in water: evidence for genotoxicity. Environ Sci Technol 40: 7394-7401.

85. Markovic Z, Todorovic-Markovic B, Kleut D, Nikolic N, Vranjes-Djuric S, et al. (2007) The mechanism of cell-damaging reactive oxygen generation by colloidal fullerenes. Biomaterials 28: 5437-5448. 
Citation: Malik P, Mukherjee TK, Singh M (2014) Biomedical Nanotoxicology and Concerns with Environment: A Prospective Approach for Merger with Green Chemistry Enabled Physicochemical Characterization. J Microb Biochem Technol S9: 001. doi:10.4172/1948-5948.S9-001

Page 14 of 14

86. Judy JD, Unrine JM, Bertsch PM (2011) Evidence for biomagnification of gold nanoparticles within a terrestrial food chain. Environ Sci Technol 45: 776-781.

87. Nurmi JT, Tratnyek PG, Sarathy V, Baer DR, Amonette JE, et al. (2005) Characterization and properties of metallic iron nanoparticles: spectroscopy, electrochemistry, and kinetics. Environ Sci Technol 39: 1221-1230.

88. Yokel RA, Macphail RC (2011) Engineered nanomaterials: exposures, hazards, and risk prevention. J Occup Med Toxicol 6: 7.

89. Chang YN, Zhang M, Xia L, Zhang J, Xing G (2012) The Toxic Effects and Mechanisms of $\mathrm{CuO}$ and ZnO Nanoparticles. Materials 5: 2850-2871.

90. Nakamura S, Mashino T (2009) Biological activities of water-soluble fullerene derivatives. Journal of Physics: Conference Series 159:012003.

91. Pauluhn J, Hahn A, Spielmann $\mathrm{H}$ (2008) Assessment of early acute lung injury in rats exposed to aerosols of consumer products: attempt to disentangle the "Magic Nano" conundrum. Inhal Toxicol 20: 1245-1262.

92. Owen R, Crane M, Grieger K, Handy R, Linkov I, Depledge M (2009) Strategic approaches for the management of environmental risk uncertainties posed by nanomaterials.UK Environment Agency (eds.). Nanotechnology: Risks and benefits, Dordrecht: Springer; 369-384.

93. Manke A, Wang L, Rojanasakul Y (2013) Mechanisms of nanoparticle-induced oxidative stress and toxicity. Biomed Res Int 2013: 942916

94. Lin PC, Lin S, Wang PC, Sridhar R (2014) Techniques for physicochemical characterization of nanomaterials. Biotechnol Adv 32: 711-726.

95. Shang L, Nienhaus K, Nienhaus GU (2014) Engineered nanoparticles interacting with cells: size matters. J Nanobiotechnology 12: 5.

96. Rivera-Gil P, Jimenez de Aberasturi D, Wulf $V$, Pelaz B, del Pino P, et al (2013) The challenge to relate the physicochemical properties of colloidal nanoparticles to their cytotoxicity. Acc Chem Res 46: 743-749.

97. Podila R, Brown JM (2013) Toxicity of engineered nanomaterials: a physicochemical perspective. J Biochem Mol Toxicol 27: 50-55.

98. Singh M (2006) Survismeter--type I and II for surface tension, viscosity measurements of liquids for academic, and research and development studies. J Biochem Biophys Methods 67: 151-161.

99. Singh M (2007) Survismeter, 2-in-1 for viscosity and surface tension measurement, an excellent invention for industrial proliferation of surface forces in liquids. Surf Rev Lett 14: 973-983.
100. Borosil Mansingh Survismeter; Singapore Patent 126089

101.Singh M (2007) Viscosities and surface tension of N,N-methylureas in aqueous solutions measured with Survismeter, a novel instrument. J Surface Sci Technology 23: 149-160.

102. Roduner E (2006) Size matters: why nanomaterials are different. Chem Soc Rev 35: 583-592.

103. Drelich L, Fang C, White CL (2002) Encyclopedia of Surface and Colloid Science 3152-3166

104. Ameta RK, Singh M (2014) SAR and DFI studies of supramolecular tetraammoniumplatinate+DNA matrix with UV/Vis spectrophotometry and physicochemical analysis at 298.15 K. J Mol Liq 190: 200-207.

105. Chandra A, Patidar V, Singh M, Kale RK (2013) Physicochemical and friccohesity study of glycine, L-alanine and L-phenylalanine with aqueous methyltrioctylammonium and cetylpyridinium chloride from $\mathrm{T}=(293.15$ to 308.15) K. J Chem Thermodyn 65: 18-28.

106. Undre SB, Singh M, Kale RK (2013) Interaction behaviour of trimesoyl chloride derived 1st tier dendrimers determined with structural and physicochemical properties required for drug designing. J Mol Liq 182: 106-120.

107. Malik P, Ameta RK, Singh M (2014) Preparation and characterization of bionanoemulsions for improving and modulating the antioxidant efficacy of natural phenolic antioxidant curcumin. Chem Biol Interact

108. Sharma K, Chauhan S (2014) Effect of biologically active amino acids on the surface activity and micellar properties of industrially important ionic surfactants. Colloids and Surfaces A: Physicochem Eng Aspects 453: 78-85.

109. Singh M, Kumar V, Kale RK (2011) Thermodynamics of philicphobic interaction shift in aqueous Tweens 20-80. International $\mathrm{J}$ of Thermodyn 14: 87-95.

110. Singh M Patel JS, Undre SB, Kale RK (2011) Densimetric and viscometric study of Gly-PVP interactions and EPA of water fitted with IMMFT at 293.15 298.15 and 303.15 K. J Mol Liq 163: 83-88.

111. Ameta RK, Singh M, Kale RK (2013) Comparative study of density, sound velocity and refractive index for (water+alkali metal) phosphates aqueous systems at $\mathrm{T}=(298.15,303.15$, and 308.15$) \mathrm{K}$. J Chem Thermodynamics 60:159-168

112. Hrncír E, Rosina J (1997) Surface tension of blood. Physiol Res 46: 319-321.

113. Vandewiele NM, Van Geem KM, Reyniers MF, Marin GB (2011) Kinetic study of the thermal rearrangement of cis- and trans-2-pinanol. J Analytical and Applied Pyrolysis 90: 187-196.
This article was originally published in a special issue, Biomaterials: Biosensors and Nano Technology handled by Editors. Dr. Valarmathi Man Thiruvanamalai, PhD, University of South Carolina, USA, Dr. Liming Ying, PhD Senior Lecturer Molecular Medicine National Heart and Lung Institute Faculty of Medicine Imperial College London, UK 